PROCEEDINGS OF THE

AMERICAN MATHEMATICAL SOCIETY

Volume 29, Number 3, August 1971

\title{
A CHARACTERIZATION OF REGULAR LOCAL RINGS
}

\section{JACOB BARSHAY}

ABstract. For a local ring, $(A, \mathfrak{M})$ of positive depth regularity is shown to be equivalent to the symmetric algebra of $\mathfrak{M}$ being torsion free.

Let $A$ be a commutative ring with unit element and $\mathfrak{A}$ an ideal of $A$. The symmetric algebra $S(\mathfrak{U})$ has been an object of study in [1], [2], and [4], particularly with regard to the questions of when it is factorial or simply a domain. In the special case where $A$ is a (Noetherian) local ring and $\mathfrak{A}=\mathfrak{M}$, its maximal ideal, Micali [1] has shown that $S(\mathfrak{M})$ is a domain if and only if $A$ is regular. It is the purpose of this note to prove the following somewhat different characterization of regular local rings.

Theorem. Let $A$ be a local ring with maximal ideal $\mathfrak{M}$, depth $A>0$. Then $A$ is regular if and only if $S(\mathfrak{M})$ is a torsion free $A$-module.

Note. An $A$-module $M$ is called torsion free if $\operatorname{ker}\left(M \rightarrow M \otimes_{A} B\right)=0$ where $B$ is the total ring of fractions of $A$.

Before proceeding with the proof, we set down some notation in the general case. Suppose that $\mathfrak{A}=\left(a_{1}, \cdots, a_{n}\right)$. Then the $A$-epimorphism $A^{n} \rightarrow \mathfrak{A}$ given by $\left(b_{1}, \cdots, b_{n}\right) \mapsto \sum_{i=1}^{n} b_{i} a_{i}$ induces an $A$-algebra epimorphism $\mu: A\left[X_{1}, \cdots, X_{n}\right] \rightarrow S(\mathfrak{H})$. If we denote the kernel of $\mu$ by $\mathfrak{Q}$, then $\mathfrak{Q}$ is the homogeneous ideal generated by all linear forms $\sum_{i=1}^{n} b_{i} X_{i}$ such that $\sum_{i=1}^{n} b_{i} a_{i}=0$.

One also has the notion of the Rees algebra, $R(\mathfrak{A})$, of the ideal $\mathfrak{A}$. This is defined as $R(\mathfrak{U})=A\left[a_{1} X, \cdots, a_{n} X\right] \subset A[X]$. The map $\mathfrak{U} \rightarrow R(\mathfrak{A})$ given by $a \mapsto a X$ induces an $A$-algebra epimorphism $\phi: S(\mathfrak{U}) \rightarrow R(\mathfrak{X})$. Finally one has the $A$-algebra epimorphism $\gamma: A\left[X_{1}, \cdots, X_{n}\right] \rightarrow R(\mathfrak{U})$ given by $X_{i} \mapsto a_{i} X$. The kernel of $\gamma$ is denoted by $\mathfrak{Q}_{\infty}$. It is the homogeneous ideal generated by all forms $f\left(X_{1}, \cdots, X_{n}\right)$ such that $f\left(a_{1}, \cdots, a_{n}\right)=0$. It is easily seen that $\boldsymbol{\gamma}=\phi \circ \mu$ and so $\operatorname{ker} \phi \approx \mathfrak{Q}_{\infty} / \mathfrak{Q}$.

These maps can be embedded in a slightly larger commutative diagram which is a useful framework for viewing things. Thus we have

Received by the editors November 17, 1970.

AMS 1969 subject classifications. Primary 1395, 1656; Secondary 1320.

Key words and phrases. Regular local ring, symmetric algebra, Rees algebra. 


$$
\begin{aligned}
& R(\mathfrak{A}) \\
& \gamma \nearrow \uparrow \phi \\
& A\left[X_{1}, \cdots, X_{n}\right] \stackrel{\mu}{\rightarrow} S(\mathfrak{A}) \\
& \downarrow \alpha \quad \downarrow \eta \\
& (A / \mathfrak{A})\left[X_{1}, \cdots, X_{n}\right] \stackrel{\lambda}{\rightarrow} S_{\boldsymbol{A} / \mathfrak{A}}\left(\mathfrak{A} / \mathfrak{A}^{2}\right)
\end{aligned}
$$

where $\alpha$ is just reduction of coefficients $\bmod \mathfrak{A}$ and $\lambda$ and $\eta$ are the obvious maps coming from the universal properties of symmetric algebras. We denote the kernel of $\alpha$ by $\mathfrak{A}^{*}$.

REMARK. Suppose that $a_{1}, \cdots, a_{n}$ is a minimal generating set for the maximal ideal $\mathfrak{M}$ in a local ring $A$. Then:

(1) $\lambda$ is an isomorphism. For if $k$ is the residue field $A / \mathfrak{M}$, then $\left[\mathfrak{M} / \mathfrak{M l}^{2}: k\right]=n$.

(2) $\mathfrak{Q}_{\infty} \subset \mathfrak{M}^{*}$ if and only if $a_{1}, \cdots, a_{n}$ are analytically independent $[3$, p. 67].

The theorem will now follow from the following lemma.

LemMA. Let $\mathfrak{A}$ be a finitely generated ideal of $A$ (not necessarily a domain) with grade $\mathfrak{A}>0$. Then ker $\phi$ is contained in the torsion submodule of $S(\mathfrak{A})$.

Proof. Suppose $\mathfrak{A}=\left(a_{1}, \cdots, a_{n}\right)$ where we can assume that $a_{n}$ is a regular element. Denote by $\mathfrak{Q}_{\infty}^{(k)}$ the forms of degree $k$ in $\mathfrak{Q}_{\infty}$. Since $\operatorname{ker} \phi \approx \mathfrak{Q}_{\infty} / \mathfrak{Q}$, it suffices to show that

$$
a_{n}^{\left(\begin{array}{c}
k \\
2
\end{array}\right)} \mathfrak{Q}_{\infty}^{(k)} \subset \mathfrak{Q} \quad \text { for all } k \geqq 1
$$

where we interpret

$$
\stackrel{(1}{2})=1 .
$$

This is done by induction on $k$ and since $\mathfrak{Q}_{\infty}^{(1)} \subset \mathfrak{Q}$, the induction is begun. Assume then that

$$
a_{n}^{\left(\begin{array}{c}
k-1 \\
2
\end{array}\right)} \mathfrak{Q}_{\infty}^{(k-1)} \subset \mathfrak{Q}
$$

and let $f \in \mathfrak{O}_{\infty}^{(\mathfrak{k})}$. One can write

$$
f=X_{1} f_{1}\left(X_{1}, \cdots, X_{n}\right)+X_{2} f_{2}\left(X_{2}, \cdots, X_{n}\right)+\cdots+X_{n} f_{n}\left(X_{n}\right)
$$

where $f_{1}, \cdots, f_{n}$ are forms of degree $k-1$. Set

$$
g=X_{1} f_{1}\left(a_{1}, \cdots, a_{n}\right)+X_{2} f_{2}\left(a_{2}, \cdots, a_{n}\right)+\cdots+X_{n} f_{n}\left(a_{n}\right) .
$$


Then $g \in \mathfrak{Q}_{\infty}^{(1)} \subset \mathfrak{Q}$. Consider now the form

$h=a_{n}^{k-1} f-X_{n}^{k-1} g=X_{1} h_{1}\left(X_{1}, \cdots, X_{n}\right)+\cdots+X_{n-1} h_{n-1}\left(X_{n-1}, X_{n}\right)$

where

$$
h_{i}\left(X_{i}, \cdots, X_{n}\right)=a_{n}^{k-1} f_{i}\left(X_{i}, \cdots, X_{n}\right)-X_{n}^{k-1} f_{i}\left(a_{i}, \cdots, a_{n}\right) .
$$

Thus every $h_{i} \in \mathfrak{Q}_{\infty}^{(k-1)}$. By the induction hypothesis

$$
a_{n}^{\left(\begin{array}{c}
k-1 \\
2
\end{array}\right)} h \in \mathfrak{Q}
$$

and so

$$
a_{n}^{k-1+\left(\begin{array}{c}
k-1 \\
2
\end{array}\right)} \cdot f=a_{n}^{\left(\begin{array}{c}
k \\
2
\end{array}\right)} f \in \mathfrak{Q},
$$

completing the induction.

Proof of Theorem. The fact that $S(\mathfrak{M})$ is torsion free, if $A$ is regular follows immediately from Micali's theorem.

Suppose now that $S(\mathfrak{M})$ is torsion free. Since depth $A>0$, i.e., grade $\mathfrak{M}>0$, we have by the Lemma, $\mathfrak{Q}_{\infty} \subset \mathfrak{Q}$. On the other hand, $\mathfrak{Q}$ $C \operatorname{ker}(\lambda \circ \alpha)=\mathfrak{M}^{*}$ by the above remark. Therefore a minimal generating set of $\mathfrak{M}$ is analytically independent. By a well-known result $[3$, p. 72 , Theorem 4$], A$ is regular.

REMARK. Since depth $A=0$ implies that every $A$-module is torsion free, the existence of nonregular local rings of depth 0 shows that the theorem cannot be extended to this case.

\section{REFERENCES}

1. A. Micali, Sur les algèbres universelles, Ann. Inst. Fourier (Grenoble) 14 (1964), fasc. 2, 33-87. MR 31 \#1275.

2. A. Micali, P. Salmon and P. Samuel, Intégreté et factorialité des algèbres symétriques, Proc. Fourth Brazilian Math. Colloq. (1963), Conselho Nacional de Pesquisas, São Paulo, 1965, pp. 61-76. MR 34 \#7556.

3. D. G. Northcott, Ideal theory, Cambridge Tracts in Math. and Math. Phys., no. 42, Cambridge Univ. Press, New York, 1953. MR 15, 390.

4. P. Samuel, Anneaux gradués factoriels et modules réflexifs, Bull. Soc. Math. France 92 (1964), 237-249. MR 32 \#160.

University of Aarhus, Aarhus, Denmark 